\title{
CHALLENGES OF LORAWAN TECHNOLOGY IN SMART CITY SOLUTIONS
}

\author{
Döníz Borsos* and Lajos Berek
}

\author{
Óbuda University, Doctoral School of Safety and Security Sciences \\ Budapest, Hungary
}

DOI: 10.7906/indecs.20.1.1

Received: 27 November 2020.

Regular article

Accepted: 20 June 2021.

\begin{abstract}
A number of technological application factors can be highlighted in smart city solutions, where small data and long-range transmission are of primary consideration. These include monitoring energy consumption, operating a smart waste management system, monitoring, and tracking traffic, having smart parking systems, monitoring public lighting, and even detecting various malfunctions. Such smart city systems use IoT technology for data collection. An essential aspect of urban solutions is to ensure the coverage of large areas, both outdoors and indoors, low energy consumption, as well as modularity and mobility. LPWAN technologies can meet these conditions. LPWAN technologies include LTE-M, NB-IoT, LoRaWAN, and Sigfox. Using LoRaWAN technology can be the right solution for these cases. As with any application, it is crucial to clarify the challenges of the application with LoRaWAN. The present study addresses the categorization of LoRaWAN devices and the challenges of technology in smart city solutions. LoRaWAN products used in smart city solutions can be grouped according to several aspects. This article deals with the creation of classifications that facilitate further testing. Smart city application challenge studies are applied to the specified categories. The challenges of the solutions cover several areas. These include technological aspects, aspects of specifications, and aspects of the nature of the application. Another area represents the information security aspects, which is, however, not addressed by this invention. Based on these aspects, the article describes the challenges of LoRaWAN technology in smart cities.
\end{abstract}

\section{KEY WORDS}

LoRa, LoRaWAN, smart city, applications, device categories

\section{CLASSIFICATION}

ACM: B.4

JEL: L63, L96 


\section{INTRODUCTION}

If we consider IoT trends, they show that there are a lot more IoT devices than predicted. Based on 2019 data, there are 9,5 billion IoT assets, compared to the estimated 8,3 billion. The number of IoT devices could reach an estimated 28 billion by 2025 [1]. Surveys show that the vast majority of these devices are related to smart city applications. Researchers among the ten most relevant trends are also among the smart city solutions in 2020 [2]. LoRaWAN is also one of the IoT, LPWAN communication technologies [3].

LoRaWAN technology is wireless, low-power, long-range radio communication that works reliably even in weak signal propagation conditions $[2,4]$. Because of these properties, they are preferred for use in smart city solutions. With the help of LoRaWAN devices, which are connected to the network, large areas, especially cities, can be easily covered. The following conclusion addresses LoRaWAN devices that are suitable for smart city solutions. The grouping of smart city solutions and areas is possible from several aspects. Six core areas can be distinguished: environment, life, people, mobility, economy, and government [5-7]. These include, for example, trackers, consumption meters, environment sensors, and parking sensors with LoRaWAN technology.

LoRaWAN communication is one of the most significant technologies used in smart city solutions. This is shown by the presence of available LoRaWAN networks in 162 countries [8]. Numerous research and development projects deal with LoRaWAN due to its topicality. An important aspect when applying a new technology is the set of requirements and challenges it has to meet and the issues it may need to solve. Each technology has its own limitations that need to be considered in such studies; this is also true for LoRaWAN. In addition, similar, competing LPWAN technologies also present challenges. The LoRa Alliance is responsible for the LoRaWAN specification, which is constantly being developed, improved, and upgraded with newer versions [8, 9]. These specifications also raise some issues. The application environment of a LoRaWAN solution (indoor, outdoor, fixed, mobile) presents additional challenges. In addition, it is important to highlight that certified LoRaWAN products can be categorized by functionality. The categories contribute to research and findings.

The structure of the article is as follows: The introduction is followed by a description of LoRaWAN technology. Next is a summary of the grouping of certified LoRaWAN products, as well as the significant challenges arising from the technology, followed by a discussion of the aspects of LoRaWAN specifications. The challenges posed by the nature of the application are also summarized. The review ends with a conclusion, which includes the possibility of additional study areas.

\section{BASICS OF LORAWAN TECHNOLOGY}

LoRaWAN network technology is based on LoRa technology developed by Semtech $[4,10]$. The first issued tool appeared around 2013-2014. The name LoRa is an abbreviation of 'long-range', which refers to the long data transmission distance that the technology is capable of. The LoRa Alliance was founded in 2015 to regulate the network operation of this technology [11]. The essence of the technology is to be able to build large-scale wireless networks, even in unfavourable field conditions, all with low energy consumption. Considering that we are talking about IoT technology, we must not forget about mobility and that it is possible to establish secure two-way communication. The standard, which is continuously updated by the LoRa Alliance, allows developers and users flexible networking and use. The essential features of LoRa and LoRaWAN communications related to the topic are highlighted. 
The long range of LoRa technology is due to the use of special frequency modulation, called chirp spread spectrum (CSS) modulation. The LoRaWAN specification defines the lower two layers of the OSI model: the physical layer and the MAC sublayer of the data link layer; and the application layer. Communication in Europe takes place at $868 \mathrm{MHz}$ and $433 \mathrm{MHz}$, but $868 \mathrm{MHz}$ is typical. Communication is two-way, with multiple channels. The devices are characterized by low power consumption; 10-year operating time is also available with a button cell. The data transmission distance in the city is a few kilometres, but it can be up to $10-15 \mathrm{~km}$ in less populated areas. The data transfer rate is relatively low, at a maximum of around $50 \mathrm{kbps}$. During communication, the messages are encrypted. The communication uses AES-128 encryption [11, 12].

The features can be summarized as:

- chirp spread spectrum (CSS) modulation,

- $868 \mathrm{MHz}$ and $433 \mathrm{MHz}$ frequencies in Europe,

- wireless radio two-way communication,

- low consumption,

- $1 \%$ transmission time,

- low consumption,

- 10-15 km bridgeable distance,

- 0,3-50 kbps data transfer rate,

- AES-128 encryption,

- star-of-stars network topology.

Network participants include end-devices, gateways, the network server, and application servers [12]. Communication between nodes and gateways takes place via LoRa. The connection between a gateway, a network server, and an application server is IP based. Nodes are usually simple devices equipped with LoRaWAN communication capability and some sensors. Gateways are connected to end-devices. There can be multiple nodes and gateways within a network. Gateways, in addition to network end-nodes, communicate with network servers. The application server and the network server are connected to each other and can be physically present on one device. The network server is responsible for monitoring and managing the network format. The application server decodes the data and displays it in a way that the user can understand. Depending on usage, multiple applications can be connected to a network. This classic network architecture is based on the 1,0 specification; other LoRaWAN specifications may result in a different network architecture [13-16].

Nodes can be divided into three classes according to their operation mode: Class A, Class B, or Class C. In all three cases, two-way communication is supported. Class A operation is optimized for consumption, with Class A devices having the lowest power consumption. Class A devices can only receive messages after they are sent, after which they open two reception windows. It is possible to send messages to the end-devices from the network server side at any time, but they can only be received after the end-device transmission period. Class $\mathrm{B}$ nodes operate with extra, scheduled reception windows in addition to the previous ones. In the case of Class $\mathrm{C}$ devices, no waiting time is to be expected; they are capable of continuous reception. This type has the highest consumption. There are uplink and downlink messages regarding the direction of data communication. An uplink message transmits from the enddevice to the network server; the downlink message's direction is the opposite. The uplink message can be an unconfirmed data message or confirmed data message [12].

Identifiers and keys are used during network communication. The most significant of these are the following. Device Address is a 32-bit identifier used to identify devices [12]. This ID is unique within each network and is part of the messages. Also worth mentioning the 
Network Session Key and the Application Session Key, which are 128-bit AES encryption keys for encrypting and decrypting data and messages [12]. The connection procedure is essential for the network operation of the nodes. This can be ABP (Activation by Personalization) or OTAA (Over-the-Air Activation) [12, 17]. In the case of ABP connection mode, a request is received from the end-devices to the network server once before starting the network operation. If the IDs and keys match, the connection is established. In OTAA connection mode, after a successful connection, a handshake connection is established between the nodes and the network server. The keys are exchanged continuously, ensuring a much more secure connection [17].

\section{GROUPING OF CERTIFIED LORAWAN PRODUCTS BY FUNCTIONALITY}

The LoRa Alliance is responsible for the certification of LoRaWAN products. The number of certified devices published on their website was 177 at the time of writing the review [18]. If LoRaWAN products are categorized, additional tests can be performed. Besides, it is necessary to describe the specific categories. There are eight categories based on the function categorization of certified LoRaWAN devices. These are as follows:

- module: a PCBA card, a basic circuit set that complies with the LoRaWAN specification and features long-range, low-power, bidirectional, wireless communication. The LoRaWAN module is not a finished product, and it is the main chip of LoRaWAN application development and finished product production [18, 19],

- development board: a printed circuit board that fundamentally contains a LoRaWAN module and the necessary additional logic associated with it, used for learning and prototyping $[18,20]$. The LoRaWAN development board can include a microcontroller or can be connected to microcontroller development boards via a typical connection line,

- sensor node: a finished product which consists of three central units: a sensor unit, a processing unit and a LoRaWAN module [18]. The sensor node can detect specific changes in the environment, react to them and transmit the processed data over the LoRaWAN network [21, 22],

- actuator node: a finished product which consists of three central units: an actuator unit, a processing unit and a LoRaWAN module [18]. The actuator can make a specific change in its environment [23], depending on the messages received on the LoRaWAN network,

- sensor interface unit: a finished product which consists of three central units: a sensor connection, a processing unit and a LoRaWAN module. The sensor interface unit connects to sensor devices [18]. It can collect data from them and then transmit the data on the LoRaWAN network after processing [24],

- meter interface unit: a finished product which consists of three central units: a standard meter connector or a standard communication interface, a processing unit and a LoRaWAN [18]. The meter interface unit can be connected to consumption meters and can collect data from them and then transmit them on the LoRaWAN network after processing,

- tracker: a finished product which consists of three central units: a locating unit, a processing unit and a LoRaWAN module [18]. The tracker is suitable for tracking persons/animals/objects, and the location data are transmitted via the LoRaWAN network [25],

- tester: a complex device that monitors and diagnoses the LoRaWAN network parameters, especially for end-devices and gateways. Another feature may be RF power testing. [18]

It is also possible to create categories according to other logic. By this logic, the ninth group would be gateways, but there is no authentication procedure for these. The formed groups can be further divided into subgroups according to further aspects. Table 1 shows the grouping of LoRaWAN products by functionality [18]. There may be devices with properties that fit several groups. In these cases, the main functionality applies. 
Table 1. Categorization of certified LoRaWAN devices [18].

\begin{tabular}{|l|c|c|c|c|c|c|c|c|}
\hline Category & Module & $\begin{array}{c}\text { Development } \\
\text { board }\end{array}$ & $\begin{array}{c}\text { Sensor } \\
\text { node }\end{array}$ & $\begin{array}{c}\text { Actuator } \\
\text { node }\end{array}$ & $\begin{array}{c}\text { Sensor } \\
\text { interface } \\
\text { unit }\end{array}$ & $\begin{array}{c}\text { Meter } \\
\text { interface } \\
\text { unit }\end{array}$ & Tracker & Tester \\
\hline $\begin{array}{c}\text { Number } \\
\text { of } \\
\text { devices }\end{array}$ & 33 & 15 & 83 & 3 & 6 & 24 & 11 & 2 \\
\hline
\end{tabular}

For a smart city, the additional findings apply to the following categories: sensor node, actuator node, sensor interface unit, measuring interface unit and tracker. These categories represent a total of 127 different products. The study does not consider devices that use LoRaWAN technology but are not on the list of certified devices. These devices can be directly or indirectly part of smart city solutions.

\section{TECHNOLOGICAL ASPECTS OF THE CHALLENGES}

Considering the challenges of LoRaWAN technology, the following three main issues can be highlighted: competing technologies, technological limitations and network operation. Closely related to these are the aspects of the specifications, which are discussed in a separate section. LoRaWAN technology belongs to LPWA networks; there are many other technologies in this area. If we highlight two major LPWAN technologies - Sigfox, and NB-IoT - the LoRaWAN technology performs below regarding the following features. The properties of the three technologies are summarized in Table 2.

Table 2. LoRaWAN, Sigfox, NB-IoT properties [3, 12, 26, 27].

\begin{tabular}{|l|l|l|l|}
\hline & \multicolumn{1}{|c|}{ LoRaWAN } & \multicolumn{1}{c|}{ Sigfox } & \multicolumn{1}{c|}{ NB-loT } \\
\hline Modulation & CSS & BPSK & QPSK \\
\hline Frequency & $868 \mathrm{MHz}-$ ISM & $868 \mathrm{MHz}-$ ISM & LTE-licensed \\
\hline Private network & Allowed & Not allowed & Not allowed \\
\hline Maximum data rate & $50 \mathrm{kbps}$ & $100 \mathrm{bps}$ & $200 \mathrm{kbps}$ \\
\hline Maximum payload & 12 bytes & 243 bytes & 1600 bytes \\
\hline Data direction & Limited bidirectional & Bidirectional & Bidirectional \\
\hline Message number & Unlimited & 40 daily & Unlimited \\
\hline Maximum range & $15 \mathrm{~km}$ & $40 \mathrm{~km}$ & $20 \mathrm{~km}$ \\
\hline $\begin{array}{l}\text { Interference } \\
\text { immunity }\end{array}$ & Very high & Very high & Low \\
\hline Encryption & AES 128 & Not supported & LTE encryption \\
\hline
\end{tabular}

The maximum data rate of LoRaWAN technology is not the lowest, at $50 \mathrm{kbps}$. The length of the payload is 1600 bytes for NB-IoT and 243 bytes for LoRaWAN. It belongs to the middle field in terms of range $(5-15 \mathrm{~km})$. It is outstanding that LoRaWAN technology is actively immune to interference and uses AES-128 encryption during communication; the adaptive data rate is implemented, and built-in positioning is used. These technological characteristics affect the areas of application. It should be emphasized that a great advantage of LoRaWAN technology, compared to similar technologies, is that private networks can be implemented with it. The private network avoids dependence on the service provider. Also, the cost of LoRaWAN network equipment and the spectrum is the lowest. 
Some of the technology limits measured above have been presented. LoRaWAN end-devices implement at least Class A operation options, in addition to which Class B and C operations are optional [13]. The operation mode is vital in the case of bi-directional communication. Class A operation results in the nodes being able to receive only for a limited time after transmission. This way, messages sent to the end-device only arrive after the transmission period of the end-device, which can reduce the application areas. This problem does not exist for Class $\mathrm{C}$ operation. Communication takes place at $868 \mathrm{MHz}$ or $433 \mathrm{MHz}$ in Europe, in the ISM band [13]. As a result, the transmission time is also limited.

The classic LoRaWAN network consists of end-devices, gateway(s), network- and application server(s) [13]. End-devices communicate with servers through gateways. It follows that the gateway is the weakest point in the network. There can be multiple gateways in a network, which increases the robustness of the communication. In addition to the implementation of private networks, it is also possible to use a service provider network and a network service.

The conclusions to be drawn for smart city solutions are as follows. This technology is not the right choice in cases:

- at high data rates,

- in case of transmission of large data,

- in case of transmission of large amounts of data,

- continuous two-way communication is essential,

- and any combination thereof.

In the case of collecting small measurement data that needs to be transmitted continuously but at infrequent intervals, this technology can be the right choice. A typical area of application is consumption metering. Unfortunately, developers also have to deal with changing user needs. Previously, it was appropriate to have meter readings taken monthly. In the case of a consumption meter with wireless communication, there are demands that users want to see the data continuously. Comparing the monthly meter readings with the continuous data provision, this seems unreasonable. Of course, the technology allows data to be provided more frequently than monthly.

\section{ASPECTS OF THE CHALLENGES RELATED TO LORAWAN SPECIFICATIONS}

The LoRa Alliance is responsible for LoRaWAN specifications and certification. Newer specifications are issued mainly for improvements, to correct bugs, and for clarifications [28] The specifications include everything that the LoRaWAN devices and networks must comply with. Compared to the 1,0 specification published in 2015, the next version (1.0.1) was released in early $2016[12,14]$. This version mostly includes spelling corrections and clarifications. The next version, 1.0.2, was released in mid-2016 with some minor changes and fixes [15]. Subsequently, specification 1.1 was released in 2017, which resulted in significant changes [15]. In addition to clarifications, it contained several functional modifications. The changes that appeared were related to the following areas: frame counters, device classes, activation modes, keys and server structure. Version 1.0.3 was released in 2018 and took over the parts for Class B devices from Specification 1.1. Previously, Class B (beacon) operations could only be encountered on a theoretical level [13].

Table 3. Number of smart city LoRaWAN devices in terms of specifications [18].

\begin{tabular}{|c|c|c|c|c|c|c|}
\hline $\begin{array}{c}\text { LoRaWAN } \\
\text { specification }\end{array}$ & $\mathbf{1 . 0}$ & $\mathbf{1 . 0 . 1}$ & $\mathbf{1 . 0}, \mathbf{1 . 0 . 2}$ & $\mathbf{1 . 0 . 2}$ & $\mathbf{1 . 0 . 3}$ & $\mathbf{1 . 1}$ \\
\hline Number of devices & 6 & 38 & 0 & 82 & 1 & 0 \\
\hline
\end{tabular}


Due to several innovations in the 1.1 specification, it is not popular with developers and operators. It is less understandable compared to the 1.0.x series [28]. If we look at the comparison tables (Table 3 and Table 4), it turns out that most of the products that meet the 1.0.2 specification are in all categories. Only one product meets the latest specification (1.0.3). Of course, the specifications are backwards compatible with each other. The conclusion is that using the latest specifications can avoid bugs and misunderstandings that can be found in older versions. It is recommended to use the 1.0.3 specification.

Table 4. Number of smart city LoRaWAN devices in terms of specifications and classification [18].

\begin{tabular}{|l|c|c|c|c|c|}
\hline Specification & $\begin{array}{c}\text { Sensor } \\
\text { node }\end{array}$ & $\begin{array}{c}\text { Actuator } \\
\text { node }\end{array}$ & $\begin{array}{c}\text { Sensor } \\
\text { interface unit }\end{array}$ & $\begin{array}{c}\text { Meter } \\
\text { interface unit }\end{array}$ & Tracker \\
\hline $\mathbf{1 . 0}$ & 5 & 0 & 0 & 1 & 0 \\
\hline $\mathbf{1 . 0 . 1}$ & 22 & 1 & 2 & 7 & 6 \\
\hline $\mathbf{1 . 0}$ and 1.0.2 & 0 & 0 & 0 & 0 & 0 \\
\hline $\mathbf{1 . 0 . 2}$ & 55 & 2 & 4 & 16 & 5 \\
\hline $\mathbf{1 . 0 . 3}$ & 1 & 0 & 0 & 0 & 0 \\
\hline $\mathbf{1 . 1}$ & 0 & 0 & 0 & 0 & 0 \\
\hline
\end{tabular}

\section{ASPECTS OF THE CHALLENGES OF THE APPLICATION NATURE}

Due to the nature of the application, we can group the devices according to several aspects. One way of grouping comes from the place of use. According to this, we can distinguish two groups: indoor devices and outdoor devices. Also, it should be mentioned that there are devices that are used both indoors and outdoors. Based on the installation of the devices, two groups can be distinguished: fixed installation and mobile. These groupings are shown in Table 5 [29].

The examined device groups cover several smart city application areas. In the sensor node category, there are mostly simple sensor devices such as a temperature meter, a humidity meter, a light sensor, an air pollution meter, and a soil moisture meter. These devices can be used both outdoors and indoors, mostly with a fixed installation. These statements are also valid for sensor interface units. The actuator node category includes mainly devices related to lighting control; they can be indoor and outdoor and have a fixed installation. The metering interface units are mostly water consumption meter-, gas meter-, and electricity meter interfaces; this means that there can be both indoor and outdoor versions with fixed installation. Trackers are mostly used for outdoor positioning, but some devices are used indoors. Trackers are specifically for mobile applications.

In the case of outdoor equipment, physical protection should be provided, and it is important to take weather factors into account. The physical protection is not specifically a LoRaWAN technology issue; the technology is less sensitive to external disturbances and environmental factors. However, for mobile applications, device movement can be a problem. In other words, the movement of the device causes a change in the radio parameters, so it is recommended to turn on the adaptive data rate (ADR) when the device is not in motion, which often happens in mobile applications [13].

As LoRaWAN end-devices are mainly battery-operated, special attention must be paid to monitoring the supply voltage and selecting the appropriate battery. Also, different antennas may be required for the same products, depending on the installation [17]. 
Table 5. Categorization of certified LoRaWAN smart city devices by applications [29].

\begin{tabular}{|c|c|c|c|c|c|}
\hline & Sensor node & $\begin{array}{l}\text { Actuator } \\
\text { node }\end{array}$ & $\begin{array}{c}\text { Sensor } \\
\text { interface unit }\end{array}$ & $\begin{array}{c}\text { Meter } \\
\text { interface } \\
\text { unit }\end{array}$ & Tracker \\
\hline $\begin{array}{c}\text { Typical } \\
\text { applications }\end{array}$ & $\begin{array}{l}\text { Temperature } \\
\text { measurement, } \\
\text { humidity } \\
\text { measurement, } \\
\text { motion } \\
\text { detection, } \\
\text { light intensity } \\
\text { measurement, } \\
\text { soil moisture } \\
\text { measurement, } \\
\text { consumption } \\
\text { measurement, } \\
\text { parking } \\
\text { sensor }\end{array}$ & $\begin{array}{l}\text { Lighting } \\
\text { control }\end{array}$ & $\begin{array}{l}\text { Temperature } \\
\text { measurement, } \\
\text { humidity } \\
\text { measurement, } \\
\text { motion } \\
\text { detection, light } \\
\text { intensity } \\
\text { measurement, } \\
\text { soil moisture } \\
\text { measurement }\end{array}$ & $\begin{array}{l}\text { Electricity } \\
\text { consumption } \\
\text { measurement, } \\
\text { water } \\
\text { consumption } \\
\text { meter, gas } \\
\text { consumption } \\
\text { meter, heat } \\
\text { quantity } \\
\text { measurement }\end{array}$ & $\begin{array}{l}\text { Person } \\
\text { tracking, } \\
\text { vehicle } \\
\text { tracking, } \\
\text { package } \\
\text { tracking, } \\
\text { animal } \\
\text { tracking }\end{array}$ \\
\hline $\begin{array}{l}\text { Indoor/ } \\
\text { Outdoor }\end{array}$ & $\begin{array}{l}\text { Indoor/ } \\
\text { Outdoor }\end{array}$ & $\begin{array}{l}\text { Mostly } \\
\text { indoor }\end{array}$ & $\begin{array}{l}\text { Indoor/ } \\
\text { Outdoor }\end{array}$ & $\begin{array}{l}\text { Indoor/ } \\
\text { Outdoor }\end{array}$ & $\begin{array}{l}\text { Mostly } \\
\text { outdoor }\end{array}$ \\
\hline Installation & Mostly fixed & Fixed & Mostly fixed & Fixed & Mobile \\
\hline
\end{tabular}

\section{CONCLUSION}

It is not news that there are many products in smart city solutions that use LoRaWAN technology. These products can be generally used for a wide variety of purposes, but especially, for data collection tasks. The technology also makes it possible to create private networks, which further increases its use. In addition to the current use cases, new directions are conceivable, which no longer provide a convenience function, but become necessary. In light of all this, there is a need for technology-related studies, measurements, and analyzes.

The study examined products that have LoRaWAN certification. The certified devices were categorized into eight groups, of which five groups were further examined: sensor node, sensor interface node, sensor interface unit, measuring interface unit and tracker. Of the application challenges, three main groups were identified: technology aspects, specification aspects and application nature aspects. The study does not address information security aspects. It can be stated that LoRaWAN technology also has several positively outstanding features compared to competing technologies. In addition, there are technology limitations (data rate, payload size, limited receive- and transmit time) that limit the scope of applicability. The results of the classification based on the LoRaWAN specification show that most of the certified devices conforming to the 1.0.2 specification. There is only one product that meets the 1.0.3 specification. A challenge is to meet the latest specifications for LoRaWAN smart city devices and complete the authentication process. For the application, the outdoor/indoor devices and the challenges of the installation method were identified. From a technological point of view, the main focus is on mobile applications, as they have to meet regularly changing environmental and radio parameters.

Future research work can be pursued in several directions. The areas currently considered can be further examined about the identified groups. Newer certified LoRaWAN products and new specifications should also be considered during reviews. The area of investigation aspects can be expanded with information security issues, examining this for network participants. By expanding the study areas and aspects, we can get a more comprehensive 
picture of the challenges related to LoRaWAN smart city solutions. If we know the challenges, recommendations can be made for LoRaWAN smart city applications.

\section{REFERENCES}

[1] IoT Analytics: IoT 2019 in Review: The 10 Most Relevant IoT Developments of the Year. http://iot-analytics.com/iot-2019-in-review, accessed $10^{\text {th }}$ January 2020,

[2] Loriot: 10 relevant IoT trends in 2020. http://loriot.io/blog/IoT-trends-2020.html, accessed $10^{\text {th }}$ January 2020,

[3] Mekkia, K.; Bajica, E.; Chaxela, F. and Meyerb, F.: A comparative study of LPWAN technologies for large-scale IoT deployment.

ICT Express 5(1), 1-7, 2019, http://dx.doi.org/10.1016/j.icte.2017.12.005,

[4] Semtech: What is LoRa? https://www.semtech.com/lora/what-is-lora, accessed $9^{\text {th }}$ January 2020,

[5] Bouzguenda, I.; Alalouch, C. and Fava, N.: Towards smart sustainable cities: A review of the role digital citizen participation could play in advancing social sustainability.

Sustainable Cities and Society 50, 1-15, 2019, http://dx.doi.org/10.1016/j.scs.2019.101627,

[6] Camero, A. and Alba, E.: Smart City and information technology: A review. Cities 93, 84-94, 2019, http://dx.doi.org/10.1016/j.cities.2019.04.014,

[7] Curzon, J.; Almehmadi, A. and El-Khatib, K.: A survey of privacy enhancing technologies for smart cities.

Pervasive and Mobile Computing 55, 76-95, 2019, http://dx.doi.org/10.1016/j.pmcj.2019.03.001,

[8] LoRa Alliance: LoRa Alliance. http://lora-alliance.org, accessed $10^{\text {th }}$ January 2020 ,

[9] Mroue, H., et al.: LoRa+: An extension of LoRaWAN protocol to reduce infrastructure costs by improving the Quality of Service.

Internet of Things 9, 1-13, 2020, http://dx.doi.org/10.1016/j.iot.2020.100176,

[10] Carvalho, D.F., et al.: A test methodology for evaluating architectural delays of LoRaWAN implementations.

Pervasive and Mobile Computing 56, 1-17, 2019, http://dx.doi.org/10.1016/j.pmcj.2019.03.002,

[11]LoRaAlliance: About LoRa Alliance. http://lora-alliance.org/about-lora-alliance, accessed $12^{\text {th }}$ January 2020 ,

[12] LoRa Alliance: LoRaWAN Specification V1.0. https://lora-alliance.org/wp-content/uploads/2020/11/2015___lorawan_specification_1r0_611_1.pdf,

[13]LoRa Alliance: LoRaWAN Specification V1.0.3. https://lora-alliance.org/wp-content/uploads/2020/11/lorawan1.0.3.pdf,

[14]LoRa Alliance: LoRaWAN Specification V1.0.1. https://lora-alliance.org/wp-content/uploads/2020/11/lorawan1.0.1final 05apr2016 1099 1.pdf

[15]LoRa Alliance: LoRaWAN Specification V1.0.2. https://lora-alliance.org/wp-content/uploads/2020/11/lorawan1 0 2-20161012 1398 1.pdf,

[16]LoRa Alliance: LoRaWAN Specification V1.1. https://lora-alliance.org/wp-content/uploads/2020/11/lorawantm_specification_v1.1.pdf,

[17] Noura, H.; Hatoum, T.; Salman, O.; Yaacoub, J-P. and Chehab, A.: LoRaWAN security survey: Issues, threats and possible mitigation techniques.

Internet of Things 12, 1-37, 2020,

http://dx.doi.org/10.1016/j.iot.2020.100303, 
[18]LoRa Alliance: Certified Products.

http://lora-alliance.org/showcase/search?is_certified=1\&categories_single $=A l l \& a=$, accessed $20^{\text {th }}$ April 2020,

[19] Muthanna, M., et al.: Cognitive control models of multiple access IoT networks using LoRa technology.

Cognitive Systems Research 65, 62-73, 2021, http://dx.doi.org/10.1016/j.cogsys.2020.09.002,

[20]Centelles, R., et al.: LoRaMoto: A communication system to provide safety awareness among civilians after an earthquake.

Future Generation Computer Systems 115, 150-170, 2021, http://dx.doi.org/10.1016/j.future.2020.07.040,

[21] Uchiyama, T.; Takamure, K.; Okuno, Y. and Sato, E.: Development of a self-powered wireless sensor node to measure the water flowrate by using a turbine flowmeter. Internet of Things 13, 1-13, 2021, http://dx.doi.org/10.1016/j.iot.2020.100327,

[22] Hashim, H.A.; Mohammed, S.L. and Gharghan, S.K.: Accurate fall detection for patients with Parkinson's disease based on a data event algorithm and wireless sensor nodes.

Measurement 156, 1-18, 2020, http://dx.doi.org/10.1016/j.measurement.2020.107573,

[23] Satpathy, S.; Sahoo, B. and Turuk, A.K.: Sensing and Actuation as a Service Delivery Model in Cloud Edge centric Internet of Things.

Future Generation Computer Systems 86, 281-296, 2018,

http://dx.doi.org/10.1016/j.future.2018.04.015,

[24] Heinssen, S., et al.: Design for reliability of generic sensor interface circuits. Microelectronics Reliability 80, 184-197, 2018, http://dx.doi.org/10.1016/j.microrel.2017.11.029,

[25]Hadwen, T.; Smallbon, V.; Zhang, Q. and D'Souza, M.: Energy efficient LoRa GPS tracker for dementia patients.

$39^{\text {th }}$ Annual International Conference of the IEEE Engineering in Medicine and Biology Society (EMBC), IEEE, Seogwipo, 2017,

http://ieeexplore.ieee.org/abstract/document/8036938, accessed $10^{\text {th }}$ November 2020,

[26] Miles, B.; Bourennane, E.M.; Boucherkha, S. and Chikhi, S.: A study of LoRaWAN protocol performance for IoT applications in smart agriculture.

Computer Communications 164, 148-157, 2020,

http://dx.doi.org/10.1016/j.comcom.2020.10.009,

[27]Ruckebusch, P.; Giannoulis, S.; Moerman, I.; Hoebeke, J. and Poorter, E.D.: Modelling the energy consumption for over-the-air software updates in LPWAN networks: SigFox, LoR $a$ and IEEE 802.15.4g.

Internet of Things 3-4, 104-119, 2018,

http://dx.doi.org/10.1016/j.iot.2018.09.010,

[28]Butun, I.; Pereira, N. and Gidlund, M.: Security Risk Analysis of LoRaWAN and Future Directions.

Future Internet 11(3), 1-22, 2019, http://dx.doi.org/10.3390/fi11010003,

[29] Andrade, R.O. and Yoo, S.G.: A Comprehensive Study of the Use of LoRa in the Development of Smart Cities.

Applied Science 19, 1-39, 2019, http://dx.doi.org/10.3390/app9224753. 